\title{
Age and mass of solar twins constrained by lithium abundance ${ }^{\star}$
}

\author{
J. D. do Nascimento $\mathrm{Jr}^{1}$, M. Castro ${ }^{1}$, J. Meléndez ${ }^{2}, \mathrm{M}$. Bazot ${ }^{2}, \mathrm{~S}$. Théado ${ }^{3}$, \\ G. F. Porto de Mello ${ }^{4}$, and J. R. De Medeiros ${ }^{1}$
}

1 Departamento de Física Teórica e Experimental, Universidade Federal do Rio Grande do Norte, CEP: 59072-970 Natal, RN, Brazil e-mail: dias@dfte.ufrn.br

2 Centro de Astrofísica da Universidade do Porto, Rua das Estrelas, 4150-762 Porto, Portugal

${ }^{3}$ Laboratoire d'Astrophysique de Toulouse et Tarbes - UMR 5572 - Université Paul Sabatier - CNRS, 14 Av. E. Belin, 31400 Toulouse, France

4 Universidade Federal do Rio de Janeiro, Observatório do Valongo, Ladeira do Pedro Antônio, 43, Rio de Janeiro, CEP: 20080-090, Brazil

Received 24 February 2009 / Accepted 27 April 2009

\section{ABSTRACT}

\begin{abstract}
Aims. We analyze the non-standard mixing history of the solar twins HIP 55459, HIP 79672, HIP 56948, HIP 73815, and HIP 100963 , to determine as precisely as possible their mass and age.

Methods. We computed a grid of evolutionary models with non-standard mixing at several metallicities with the Toulouse-Geneva code for a range of stellar masses assuming an error bar of $\pm 50 \mathrm{~K}$ in $T_{\text {eff }}$. We choose the evolutionary model that reproduces accurately the observed low lithium abundances observed in the solar twins.

Results. Our best-fit model for each solar twin provides a mass and age solution constrained by their Li content and $T_{\text {eff }}$ determination. HIP 56948 is the most likely solar-twin candidate at the present time and our analysis infers a mass of $0.994 \pm 0.004 M_{\odot}$ and an age of $4.71 \pm 1.39 \mathrm{Gyr}$.

Conclusions. Non-standard mixing is required to explain the low $\mathrm{Li}$ abundances observed in solar twins. Li depletion due to additional mixing in solar twins is strongly mass dependent. An accurate lithium abundance measurement and non-standard models provide more precise information about the age and mass more robustly than determined by classical methods alone.
\end{abstract}

Key words. stars: fundamental parameters - stars: horizontal-branch - stars: abundances - stars: evolution - stars: interiors Sun: fundamental parameters

\section{Introduction}

Lithium is easily destroyed by nuclear burning in stellar interiors at temperatures above $2.4 \times 10^{6} \mathrm{~K}$ and its surface abundance in main-sequence stars indicates the depth of mixing below their photospheres. As in the Sun, the amount of Li depletion in solar twins is sensitive to microscopic diffusion, and some extramixing process is required to explain the low observed $\mathrm{Li}$ abundances, indicating that they also share with the Sun a similar mixing history. Standard solar models predict that the Sun has a convective envelope whose mass is only $2 \%$ of the total mass and where the base temperatures are generally too low to destroy lithium. The solar Li problem is the long-standing conflict between the observed photospheric Li depletion of the Sun by 2.21 dex (Grevesse \& Sauval 1998) and the low theoretically predicted depletion of stellar evolution models based only on the standard mixing-length prescription.

Many discoveries have been made that have changed our understanding of our central G2 type star. Until a few years ago, the Sun was thought to be lithium-poor by a factor of 10 compared to similar one-solar-mass solar-type disk stars (Lambert \& Reddy 2004), which led to the suggestion that the Sun is peculiar in its

\footnotetext{
* The models are only available in electronic form at the CDS via anonymous ftp to cdsarc.u-strasbg.fr (130.79.128.5) or via http://cdsweb.u-strasbg.fr/cgi-bin/qcat?J/A+A/501/687 or via http://andromeda.dfte.ufrn.br
}

Li abundance and therefore of dubious value for calibrating nonstandard models of Li depletion. However, current studies have shown that the Sun seems to be typical when compared to solar twins instead of merely solar-type stars, and that solar twins also have low Li abundances (Meléndez \& Ramírez 2007; Pasquini et al. 2008).

The history of internal mixing and Li depletion of each star is probably dependent of the evolution of its rotational history and the changes in the convective envelope over time. These parameters are mass and metallicity dependent, and eventual differences in them, from birth, may exist between very similar stars and yet be too small to be measured observationally with conventional techniques. Therefore, the detailed internal modeling of solar twin stars, already established to be extremely similar to the Sun in many astrophysical properties, is potentially a powerful tool for boosting our comprehension of the complex evolution of the $\mathrm{Li}$ abundance in low-mass stars.

We know that the Sun is not unique in being a planet host. However, the Sun is still unique in the sense that no other truly solar-like planetary system has been detected to date, although some solar twins, which do not seem to have either hot Jupiters or other giant planets in their habitable zones (e.g., HIP 79672 (18 Sco), HIP 56 948; Meléndez \& Ramírez 2007), are excellent candidates for hosting Earth-like planets, and therefore they should be analyzed in detail both from the theoretical and observational point of view. The quest to identify 
stellar analogues to the Sun or true solar twins has continued for a long time (Cayrel de Strobel 1996; Porto de Mello $\&$ da Silva 1997). High resolution, high signal-to-noise data analyzed by Meléndez \& Ramírez (2007) demonstrated that HIP 56948 is the most likely solar twin known to date both in terms of stellar parameters and chemical composition, including a low lithium abundance. The depletion of $\mathrm{Li}$ in field solar-analog $\mathrm{G}$ dwarf stars appears to highlight our limited understanding of the physics acting in the interiors of stars. To explain the unexpected main-sequence Li depletion of $\mathrm{F}$ and early G-type stars, a number of models and extra-mixing processes have been proposed, including mass loss (Swenson \& Faulkner 1992), diffusion (Michaud 1986; Chaboyer et al. 1995), meridional circulation (Charbonnel \& Talon 1999, and references therein), angular momentum loss, and rotationally driven mixing (Schatzman \& Baglin 1991; Vauclair 1991; Pinsonneault et al. 1992; Deliyannis \& Pinsonneault 1997; Charbonnel \& do Nascimento 1998), gravity waves (García López \& Spruit 1991; Montalban \& Schatzmann 2000), tachocline (Brun et al. 1999; Piau et al. 2003), and combinations of these (Charbonnel \& Talon 2005). In this work, we present evolutionary solar-like models with microscopic diffusion and rotation-induced mixing in the radiative interior. This mixing due to the meridional circulation with a feedback effect of the $\mu$-currents is described in Zahn (1992), Vauclair \& Théado (2003), and Théado \& Vauclair (2003a). We also introduce macroscopic motions caused by the tachocline (Richard et al. 2004), where the shear turbulence mixes and homogenizes with the convective zone the material transported by the meridional circulation. The diffusioncirculation coupling allowed both sides of the Li-dip to be reproduced (Théado \& Vauclair 2003b) and the addition of a tachocline provides very good agreement with helioseismic observations in solar models (Richard et al. 2004). Since Li is probably an indicator of the complex processes that occurred in the past between the stellar external layers and the hotter interior, its abundance can be used to identify true solar twins, because solar twins are expected to share not only the present parameters of the Sun but also the essential aspects of its evolution.

The present working sample is described in Sect. 2, where we redetermine the evolutionary status and individual masses of the sample by using the HIPPARCOS parallaxes and by comparing the observational Hertzsprung-Russell diagram with evolutionary tracks. In Sect. 3, we describe the evolutionary models with non-standard physics. In Sect. 4, we present the lithium abundance main features and compare the observations with theoretical predictions. Finally, our conclusions are outlined in Sect. 5.

\section{Solar twin sample}

Our analysis is based on the three solar twins, HIP 55459, HIP 79672, and HIP 100 963, analyzed by Takeda et al. (2007), and the four solar twins, HIP 55459 , HIP 79672, HIP 56948 , and HIP 73815 studied by Meléndez \& Ramírez (2007). Two stars, HIP 55459 and HIP 79672, are present in both samples. Thus, the sample consists of 5 solar twins. Following the same procedure as do Nascimento et al. (2000), we used the new HIPPARCOS trigonometric parallax measurements (van Leeuwen 2007) to locate precisely the objects in the HR diagram. Intrinsic absolute magnitudes $M_{\mathrm{V}}$ were derived from the parallaxes and the $m_{\mathrm{V}}$ magnitudes were those given by HIPPARCOS. We computed the stellar luminosity and the associated error from the $\sigma$ error in the parallax. The uncertainties in luminosity lower than \pm 0.1 had an effect of \pm 0.03 in the determination of the masses.

In Takeda et al. (2007), HIP 55459, HIP 79672, and HIP 100963 are taken from the sample of 118 solar-analogue stars, observed by HIDES (Izumiura 1999) at the coudé focus of the $188 \mathrm{~cm}$ reflector of Okayama Astrophysical Observatory (OAO). These three stars are G main-sequence stars, which are considered to be solar twins. Their Li abundance is however a factor of 3 to 4 higher than solar. Meléndez \& Ramírez (2007) presented their data and results for HIP 55459, HIP 79672, HIP 56 948, and HIP 73815 observed with the 2dcoudé spectrograph (Tull et al. 1995) on the 2.7-m Harlan J. Smith Telescope at McDonald Observatory. HIP 56948 and HIP 73815 are considered by these authors to be the most similar stars to the Sun ever found, because their Li abundances are as low as solar. Atmospheric parameters of all of these stars are in excellent agreement with those of the Sun, lower than $1.3 \%$ for the $T_{\text {eff }}$ and a few hundredths dex for $\log \left(L / L_{\odot}\right)$ and $[\mathrm{Fe} / \mathrm{H}]$.

Two observed trends provide a first order explanation of the Li abundance in these solar twins. HIP 55459 and HIP 79672 are indeed found to be slightly more massive than the Sun by $\approx 3-4 \%$ (Takeda et al. 2007; Meléndez \& Ramírez 2007), which could explain the overabundance of Li compared to solar lithium, the external convective zone being shallower. The other trend concerns the age of the stars since HIP 56948 and HIP 73815 are older than the Sun and exhibit a stronger Li-depletion. The sample of solar-twins is summarized in Table 1.

\section{Stellar evolutionary models}

For the purposes of this study, stellar evolution calculations were computed with the Toulouse-Geneva stellar evolution code TGEC (Hui-Bon-Hoa 2008). Details of the physics of these models can be found in Richard et al. (1996, 2004), do Nascimento et al. (2000), and Hui-Bon-Hoa (2008). We describe the input standard physics, non-standard processes, diffusion, and rotation-induced mixing added in the models.

\subsection{Input physics}

We used the OPAL2001 equation of state by Rogers \& Nayfonov (2002) and the radiative opacities by Iglesias \& Rogers (1996), completed with the low temperature atomic and molecular opacities by Alexander \& Ferguson (1994). The nuclear reactions are from the analytical formulae of the NACRE (Angulo et al. 1999) compilation, taking into account the three $p p$ chains and the CNO tricycle with the Bahcall \& Pinsonneault (1992) screening routine. Convection is treated according to the Böhm-Vitense (1958) formalism of the mixing length theory with a mixing length parameter $\alpha=l / H_{\mathrm{p}}=1.74$, where $l$ is the mixing length and $H_{\mathrm{p}}$ the pressure height scale. For the atmosphere, we use a gray atmosphere following the Eddington relation, which is a good approximation for main-sequence solar-type stars (VandenBerg et al. 2008).

The abundance variations in the following chemical species are computed individually in the stellar evolution code: $\mathrm{H}, \mathrm{He}$, $\mathrm{C}, \mathrm{N}, \mathrm{O}, \mathrm{Ne}$, and $\mathrm{Mg}$. Both $\mathrm{Li}$ and $\mathrm{Be}$ are treated separately only as a fraction of the initial abundance. The heavier elements are gathered in a mean species $\mathrm{Z}$. The initial composition follows the Grevesse \& Noels (1993) mixture with an initial helium abundance $\mathrm{Y}_{\text {ini }}=0.268$. We chose to use the "old" abundances of Grevesse \& Noels (1993) instead of the "new" 
Table 1. Parameters of the observed solar twins.

\begin{tabular}{cccccccl}
\hline \hline Name & $\begin{array}{c}T_{\text {eff }} \\
(\mathrm{K})\end{array}$ & $\left(L / L_{\odot}\right)$ & {$[\mathrm{Fe} / \mathrm{H}]$} & $\log N(\mathrm{Li})$ & $\begin{array}{c}\text { Age } \\
(\mathrm{Gyr})\end{array}$ & $\begin{array}{c}\text { Mass } \\
\left(M_{\odot}\right)\end{array}$ & Source $^{a}$ \\
\hline HIP 55 459 & $5812 \pm 50$ & $1.081 \pm 0.069$ & 0.066 & $1.58 \pm 0.07$ & $3.80_{-2.10}^{+2.51}$ & $1.03_{-0.04}^{+0.05}$ & $(\mathrm{i})^{*}$ \\
$(\mathrm{HD} 98618)$ & $5837 \pm 36$ & $1.13 \pm 0.11$ & 0.030 & $1.55 \pm 0.08$ & $4.7 \pm 1.0$ & $1.03 \pm 0.03$ & $(\mathrm{~g})^{*}$ \\
& $5812 \pm 44$ & $(\ldots)$ & $0.03 \pm 0.03$ & $(\ldots)$ & $4.9 \pm 2.9$ & $1.040 \pm 0.150$ & $(\mathrm{c})$ \\
& $5843 \pm 30$ & $(\ldots)$ & $0.05 \pm 0.03$ & $1.57 \pm 0.09$ & $4.3 \pm 0.9$ & $1.02 \pm 0.03$ & $(\mathrm{e})$ \\
HIP 79 672 & $5762 \pm 50$ & $1.043 \pm 0.027$ & 0.039 & $1.63 \pm 0.07$ & $4.57_{-2.87}^{+0.33}$ & $1.02_{-0.02}^{+0.03}$ & $(\mathrm{i})^{*}$ \\
$(18$ Sco $)$ & $5853 \pm 36$ & $1.06 \pm 0.09$ & 0.040 & $1.63 \pm 0.08$ & $3.4 \pm 1.0$ & $1.04 \pm 0.03$ & $(\mathrm{~g})^{*}$ \\
& $5789 \pm 30$ & $(\ldots)$ & $0.05 \pm 0.06$ & $(\ldots)$ & $(\ldots)$ & $(\ldots)$ & $(\mathrm{a})$ \\
& 5823 & $(\ldots)$ & 0.03 & $(\ldots)$ & $(\ldots)$ & $(\ldots)$ & $(\mathrm{b})$ \\
& $5791 \pm 44$ & $(\ldots)$ & $0.03 \pm 0.03$ & $(\ldots)$ & $4.7 \pm 2.7$ & $0.980 \pm 0.130$ & $(\mathrm{c})$ \\
& $5817 \pm 30$ & $(\ldots)$ & $0.02 \pm 0.03$ & $1.63 \pm 0.09$ & $4.0 \pm 0.4$ & $1.02 \pm 0.03$ & $(\mathrm{e})$ \\
HIP 100 963 & $5779 \pm 50$ & $0.968 \pm 0.043$ & -0.002 & $1.72 \pm 0.07$ & $5.13_{-2.99}^{+0.000}$ & $1.00_{-0.02}^{+0.03}$ & $(\mathrm{i}) *$ \\
& 5794 & $(\ldots)$ & $(\ldots)$ & $(\ldots)$ & $(\ldots)$ & $(\ldots)$ & $(\mathrm{d})$ \\
& 5803 & $(\ldots)$ & -0.004 & 1.68 & $(\ldots)$ & $(\ldots)$ & $(\mathrm{j})$ \\
HIP 56 948 & $5785 \pm 36$ & $1.15 \pm 0.14$ & 0.020 & $1.08 \pm 0.13$ & $5.8 \pm 1.0$ & $1.00 \pm 0.03$ & $(\mathrm{~g})^{*}$ \\
& 5785 & $(\ldots)$ & $(\ldots)$ & $(\ldots)$ & $(\ldots)$ & $(\ldots)$ & $(\mathrm{d})$ \\
& 5701 & $(\ldots)$ & -0.15 & $(\ldots)$ & 9.6 & $0.98 \pm 0.05$ & $(\mathrm{f})$ \\
& 5791 & $(\ldots)$ & 0.020 & 1.13 & $(\ldots)$ & $(\ldots)$ & $(\mathrm{j})$ \\
HIP 73 815 & $5810 \pm 36$ & $1.21 \pm 0.14$ & 0.010 & $0.95 \pm 0.20$ & $6.3 \pm 1.0$ & $1.00 \pm 0.03$ & $(\mathrm{~g})^{*}$ \\
& 5759 & $(\ldots)$ & -0.05 & $(\ldots)$ & $(\ldots)$ & $(\ldots)$ & $(\mathrm{h})$ \\
\hline
\end{tabular}

${ }^{a}$ The sources of the observations are: (a) Porto de Mello \& da Silva (1997), (b) Luck \& Heiter (2005), (c) Valenti \& Fisher (2005), (d) Masana et al. (2006), (e) Meléndez et al. (2006), (f) Holmberg et al. (2007), (g)* Meléndez \& Ramírez (2007), (h) Robinson et al. (2007), (i)* Takeda et al. (2007), (j) Takeda \& Tajitsu (2009). The observations used in this work are represented by *. ${ }^{b}$ Takeda et al. (2007) give no estimation for the upper error of the age of HIP 100963.

mixture of Asplund et al. (2005). This choice is motivated by the disagreement between these new abundances and the helioseismic inversions for the sound-speed profile, the surface helium abundance, and the convective zone depth. Furthermore, Caffau et al. (2009) revised the solar metallicity using 3D hydrodynamical models to $Z=0.0156$ and $Z / X=0.0213$. These values are closer to those of Grevesse \& Noels (1993).

\section{Diffusion and rotation-induced mixing}

The microscopic diffusion is computed with the atom test approximation. All models include gravitational settling with diffusion coefficients computed as in Paquette et al. (1986). Radiative accelerations are not computed here, since we focus only on solar-type stars where their effects are small when mixing is taken into account (Turcotte et al. 1998; Delahaye \& Pinsonneault 2005). Rotation-induced mixing is computed as described in Théado \& Vauclair (2003a). This prescription is an extension of the approach of Zahn (1992) and Maeder \& Zahn (1998), and introduces the feedback effect of the $\mu$-currents in the meridional circulation, caused by the diffusion-induced molecular weight gradients. It introduces two free parameters in the computations $\left(\mathrm{C}_{\mathrm{h}}\right.$ and $\alpha_{\text {turb }}$ : cf. Eq. (20) of Théado \& Vauclair 2003a).

The evolution in the rotation profile follows the Skumanich's law (Skumanich 1972) with an initial surface rotation velocity on the ZAMS equal to $V_{i}=100 \mathrm{~km} \mathrm{~s}^{-1}$. Other prescriptions were tested by other authors to model the lithium destruction. Charbonnel \& Talon (1999) and Palacios et al. (2003) included angular momentum transport induced by mixing. However, since a rotation-induced mixing alone cannot account for the flat rotation profile inside the Sun, these authors later introduced the possible effect of internal gravity waves triggered at the bottom of the convective zone (e.g., Talon \& Charbonnel 2005), which allows us to reproduce the hot side of the Li-dip. Other authors suggested that the internal magnetic field is more important than internal waves in transporting angular momentum (Gough \& McIntyre 1998). In any case, when applied to the solar case, all of these prescriptions are able to reproduce the lithium depletion observed in the Hyades, and the results are ultimately quite similar (Talon \& Charbonnel 1998; Theado \& Vauclair 2003b).

We also include a shear layer below the convective zone, which is treated as a tachocline (see Spiegel \& Zahn 1992). This layer is parameterized with an effective diffusion coefficient that decreases exponentially downwards (see Brun et al. 1998; Brun et al. 1999; Richard et al. 2004):

$D_{\text {tacho }}=D_{\text {bcz }} \exp \left(\ln 2 \frac{r-r_{\mathrm{bcz}}}{\Delta}\right)$

where $D_{\text {bcz }}$ and $r_{\mathrm{bcz}}$ are the value of $D_{\text {tacho }}$ at the bottom of the convective zone and the radius at this location respectively, and $\Delta$ is the half width of the tachocline. Both $D_{\text {bcz }}$ and $\Delta$ are free parameters and the absolute size of the tachocline (i.e., $\Delta / R_{*}$ where $R_{*}$ is the radius of the star) is supposed to be constant during the evolution.

\subsection{Models and calibration}

We calculated evolutionary models of different masses from the zero-age main sequence (ZAMS) to the end of the hydrogen exhaustion in the core. Our evolutionary models were calibrated to match the observed solar effective temperature and luminosity at the solar age. The calibration method of the models is based on the Richard et al. (1996) prescription: for a $1.00 M_{\odot}$ star, we adjusted the mixing-length parameter $\alpha$ and the initial helium abundance $Y_{\text {ini }}$ to reproduce the observed solar 

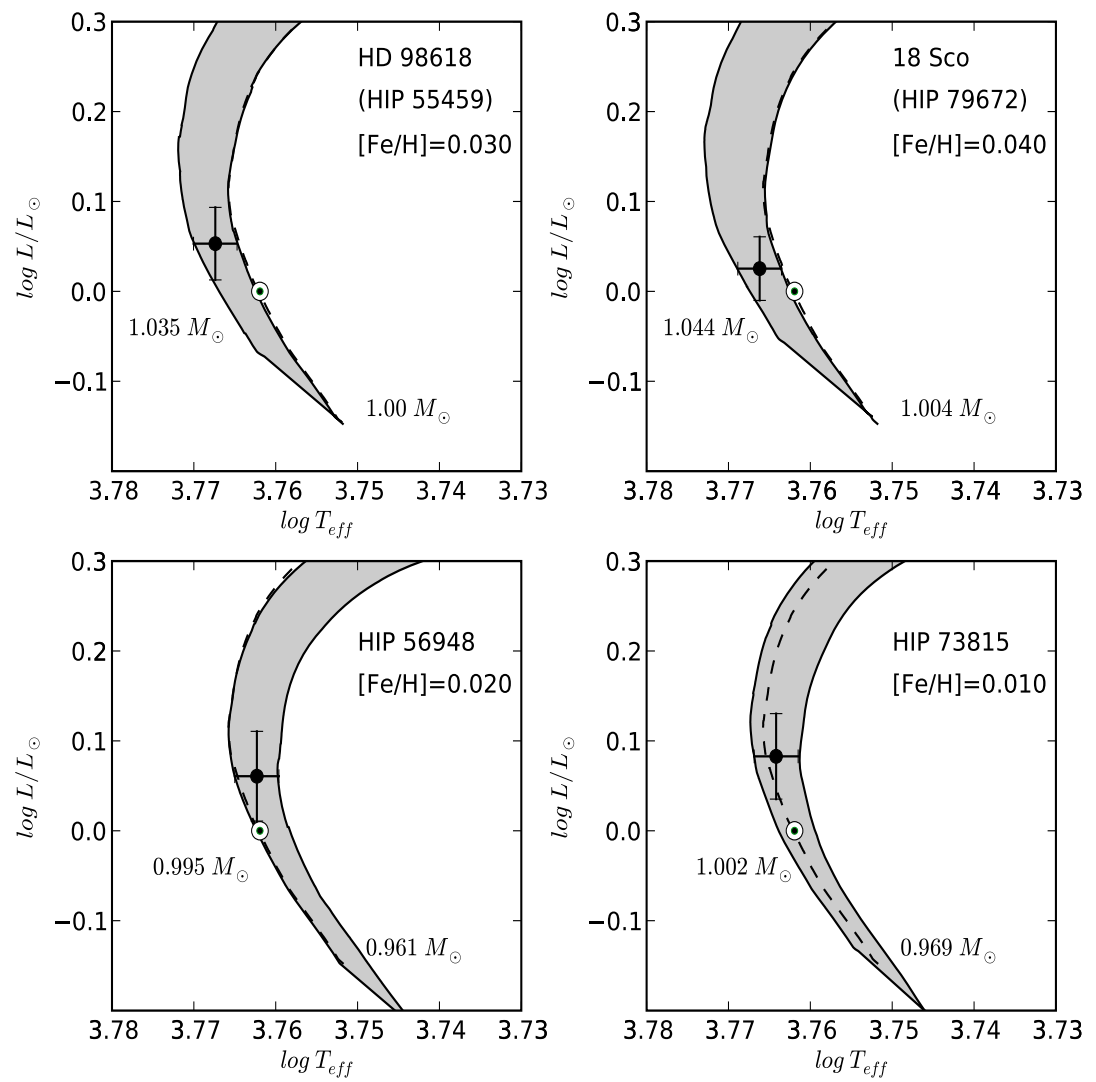

Fig. 1. Solar twins stars observed by Mélendez \& Ramírez (2007) in the Hertzsprung-Russell diagram. Luminosities and related errors have been derived from the Hipparcos parallaxes. The typical errors on $T_{\mathrm{eff}}$ are indicated in Table 1 . The shaded zone represents the range of masses in TGEC models (the maximum and the minimum masses are indicated) limited by the 1- $\sigma$ observational error bars.

luminosity and radius at the solar age. The observed values that we used are those of Richard et al. (2004), i.e., $L_{\odot}=3.8515 \pm$ $0.0055 \times 10^{33} \mathrm{erg} \mathrm{s}^{-1}, R_{\odot}=6.95749 \pm 0.00241 \times 10^{10} \mathrm{~cm}$, and age $\odot_{\odot}=4.57 \pm 0.02$ Gyr. For our best-fit solar model, we obtained $L=3.8499 \times 10^{33} \mathrm{erg} \mathrm{s}^{-1}$ and $R=6.95938 \times 10^{10} \mathrm{~cm}$ at an age $=4.576 \mathrm{Gyr}$.

The free parameters of the rotation-induced mixing determine the efficiency of the turbulent motions. They are adjusted to produce a mixing that is both: 1) efficient and deep enough to smooth the helium gradient below the outer convective zone; 2) weak and shallow enough to avoid the destruction of Be. Following Grevesse \& Sauval (1998), the Be abundance of the Sun is $\log N(\mathrm{Be})=1.40 \pm 0.09$, and Balachandran \& Bell (1998) showed that, after correcting for the continuous opacity in the ultraviolet region of the spectrum, solar beryllium is not depleted at all with respect to the meteoritic value. However, the source of "extra UV" opacity has never been identified and it is justified by assuming that the $\mathrm{OH}$ lines in the UV and the IR are both formed in LTE. We obtained a slight Be destruction by a factor of 1.25 with respect to the meteoritic value, which is well within the error in the determination of the solar Be abundance.

The calibration of the tachocline allows us to reach the solar lithium depletion $(\log N(\mathrm{Li})=1.10 \pm 0.10$, e.g., Grevesse \& Sauval 1998), and for our best-fit solar model we obtained $\log N(\mathrm{Li})=1.13$. We also checked that the sound velocity profile of our best-fit model is consistent with that deduced from helioseismology inversions by Basu et al. (1997). Our calibration is in an excellent agreement with helioseismology, more accurately than $1 \%$ for most of the star, except in the deep interior, where it reaches $1.5 \%$. The input parameters for the other masses are the same as for the $1.00 M_{\odot}$ model.

\section{Results and discussion}

Lithium is a key element because it is easily destroyed in stellar interiors. Its abundance indicates the amount of internal mixing in the stars and its destruction is strongly mass and age dependent. Nowadays, it is well established on empirical arguments that a non-standard mixing mechanism must be operating to explain the low Li abundances in solar-type stars.

For each star in our sample, we have first computed a grid of models as described above with a mass step of $0.001 M_{\odot}$ and with masses limited by the $1-\sigma$ error bars in the HR diagram (Figs. 1 and 2). The metallicity of the models matches that observed for each solar twin. This first comparison in the HR diagram between the observations of solar twins and the models gives us an estimation of their mass and age, and of the precision of our method. The masses inferred are within the range expected for the mass of a solar twin ( $\pm 5 \%$ of the solar mass). The precision of the mass determination is directly linked to the precision of the $T_{\text {eff }}$ estimations from the observations. In the case of stars observed by Mélendez \& Ramírez (2007), the error associated with the effective temperature is $\pm 36 \mathrm{~K}$, and on average we obtain a spread in masses $\langle\Delta M\rangle \sim 0.036 M_{\odot}$, within the limits of the $T_{\text {eff }}$ errors. For the stars studied by Takeda et al. (2007), the error associated with $T_{\text {eff }}$ is $\pm 50 \mathrm{~K}$ and the average range of masses is $\langle\Delta M\rangle \sim 0.056 M_{\odot}$. This first estimation is 
quite satisfactory, but by using the observed Li abundance we are able to reach even higher precision.

Figures 3 and 4 show the lithium destruction of our models and the observed abundance of each solar twin as a function of effective temperature. The error associated with $\log N(\mathrm{Li})$ is about 0.1 dex. As in Figs. 1 and 2, we plot a zone corresponding to the grid of models with masses limited by the 1- $\sigma$ error bars. For each star, this infers a $\Delta \mathrm{M}$ that is even smaller, $\langle\Delta M\rangle \sim 0.008 M_{\odot}$ for Mélendez \& Ramírez (2007) stars, and $\langle\Delta M\rangle \sim 0.012 M_{\odot}$ for Takeda et al. (2007) stars. We also computed a model track that passes through the observed position of the star in the $T_{\text {eff }}-\mathrm{Li}$ diagram. It provides the most probable modeling of the observed star, and the values of our mass and age estimations.

The uncertainties in the external parameters of our models are difficult to evaluate and have different sources. The use of a more sophisticated atmosphere model than the 1-D grey atmosphere computations or a changed in the internal physics could modify the derived effective temperature. However, the present models and their parameters were calibrated to account precisely for the solar observed external parameters, i.e., the solar soundvelocity profile and for the solar lithium depletion, with a solar model. The uncertainties that we deduced for the mass and age estimations were calculated for the values of mass and age from the two extreme models in the diagrams Li- $T_{\text {eff }}$. These uncertainties are lower limits since only two parameters are being sampled. In this context, the original result of our analysis is then that the masses and $\log N(\mathrm{Li})$ values associated with a nonstandard model at a given $T_{\text {eff }}$ provide us with a mass solution that is more precise than a mass determined based only on the HR diagram position.

\subsection{Comparison with previous results}

HIP 55459 was previously observed by Valenti \& Fisher (2005) and Meléndez et al. (2006). The values that we used in our analysis are those from Takeda et al. (2007) and Meléndez \& Ramírez (2007) (see Table 1). The Li abundance appears to be enhanced compared to the Sun, although from observations and comparison with evolutionary models, the star appears to be significantly more massive than the Sun with an age between 4.27 $\pm 1.94 \mathrm{Gyr}$ and $5.77 \pm 0.81$ Gyr. Our estimated mass, of $1.018 \pm 0.007 M_{\odot}$ comparing with Takeda et al. (2007) and 1.013 $\pm 0.005 M_{\odot}$ comparing with Meléndez \& Ramírez (2007), is lower than all other determinations (see Table 2). However, in the determination by comparison with the observations of Takeda et al. (2007), we were unable to obtain an evolutionary track consistent with the hot side of the error bar, as seen in the left upper box of Fig. 4.

HIP 79672 is the oldest star that is considered to be a solar twin. It was observed by Porto de Mello \& da Silva (1997), Valenti \& Fisher (2005), Luck \& Heiter (2005), and Meléndez et al. (2006). In this work, we have used the values from both Takeda et al. (2007) and Meléndez \& Ramírez (2007) (see Table 1$)$. The $\log N(\mathrm{Li})$ of $18 \mathrm{Sco}$ provides us with a picture of a star with a mass between $0.7 \%$ and $1.5 \%$ higher than the Sun. The age is difficult to estimate because we found $2.89 \pm 1.09 \mathrm{Gyr}$ with the observations of Takeda et al. (2007) and 5.03 $\pm 1.29 \mathrm{Gyr}$ with Meléndez \& Ramírez (2007) (see Table 2). These uncertainties in the present estimations originate in the discrepancies between Meléndez \& Ramírez (2007) and Takeda et al. (2007), especially for the effective temperature estimation. In both cases, we can observe that all lithium determinations are in close agreement. Thus, the principal discrepancy between mass and age
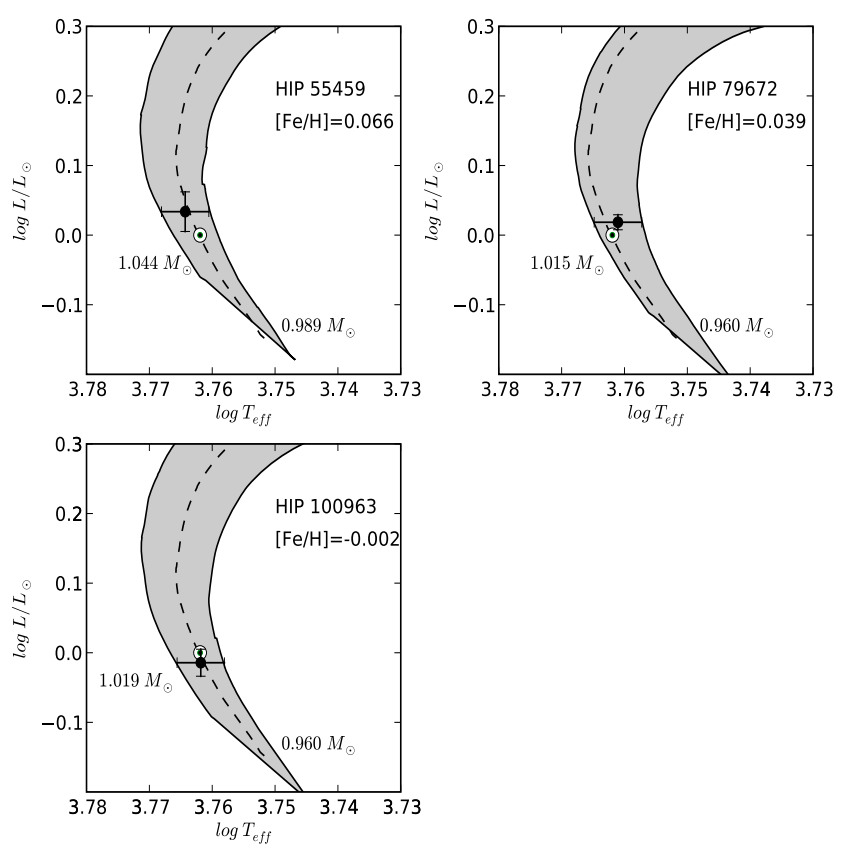

Fig. 2. Solar twins observed by Takeda et al. (2007), the caption is as in Fig. 1.

estimated from the observations of Meléndez \& Ramírez (2007) and Takeda et al. (2007) comes from the $T_{\text {eff }}$ difference.

HIP 100963 was observed by Masana et al. (2006) and we used the observations of Takeda et al. (2007) (see Table 1). The estimated age is around 5.13 Gyr. Our estimation obtained by comparing with this most recent observation implies a stellar mass very close to the solar value $\left(M=0.998 \pm 0.006 M_{\odot}\right)$ but younger (between 2.01 and $3.80 \mathrm{Gyr}$ ), which is consistent with the observed lithium abundance (see Table 2).

HIP 56948 is currently the most likely solar twin. Observations of this star were completed by Holmberg et al. (2007) and Masana et al. (2006). The latest observations from Meléndez \& Ramírez (2007) were used in this work (see Table 1). We confirm that HIP 56948 appears to be an excellent solartwin candidate of mass $M=0.994 \pm 0.004 M_{\odot}$ and age $=4.71 \pm$ $1.39 \mathrm{Gyr}$, which is even closer to the solar value than the age determined by Meléndez \& Ramírez (2007) (5.8 \pm 1.0 Gyr; see Table 2). If the mixing processes involved in the interior are the same in both the Sun and HIP 56948, the fact that these two stars have roughly the same Li content, and that HIP 56948 is slightly less massive, suggests that the latter is slightly younger.

HIP 73815 was analyzed by Robinson et al. (2007). We used the measurements of Meléndez \& Ramírez (2007) (see Table 1). The abundance $\log N(\mathrm{Li})$ is consistent with a solar twin of $0.992 \pm 0.005 M_{\odot}$ and an age of $5.76 \pm 1.13 \mathrm{Gyr}$ (see Table 2).

Takeda \& Tajitsu (2009) proposed new determinations of the external parameters of HIP 56 948, HIP 79672, and HIP 100963 (see Table 1). The new determination of $T_{\text {eff }}$ for HIP 79672 is much closer to that of Meléndez \& Ramírez (2007). A new estimation should reduce the discrepancies that we found between the two determinations of both mass and age. The new determination of $T_{\text {eff }}$ for HIP 100963 is close to the old value, and should not change our estimation of mass and age significantly. Concerning HIP 56948, these determinations are close to those of Meléndez \& Ramírez (2007), and a study that applies our method with these observations should confirm our results for this solar twin. Our results are summarized in Table 2 and Fig. 5. 

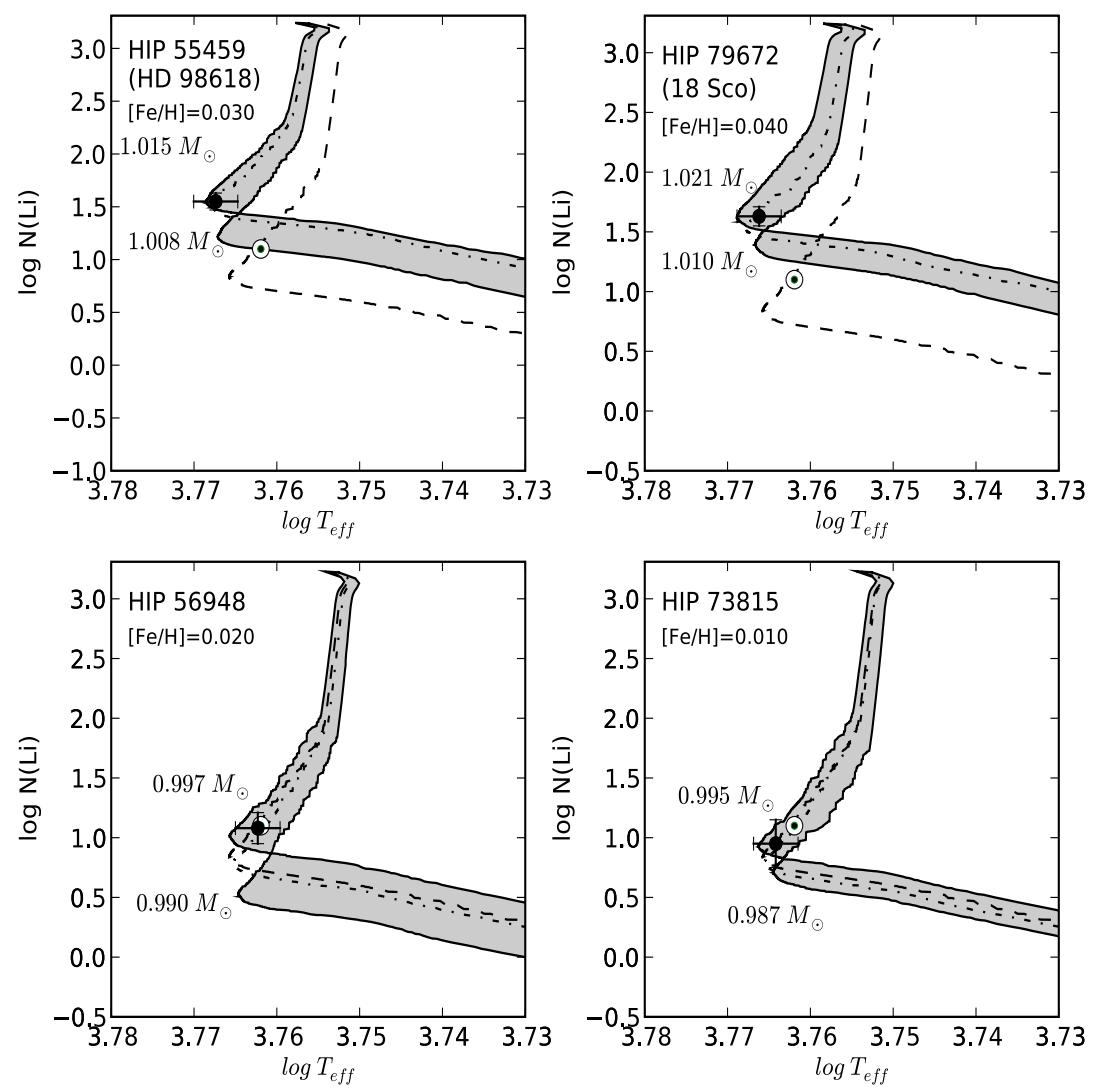

Fig. 3. Solar twins observed by Mélendez \& Ramírez (2007): lithium destruction along the evolutionary tracks as a function of the effective temperature. The shaded zone represents the range of masses of TGEC models (the maximum and the minimum masses are indicated) limited by the $1-\sigma$ observational error bars. A model (dot-dashed lines) of $1.013 M_{\odot}$ (HIP 55 459), $1.015 M_{\odot}$ (HIP 79672 ), $0.994 M_{\odot}$ (HIP 56 948 ), and $0.992 M_{\odot}$ (HIP 73 815) passes through the observed point. The positions of the solar twin, the Sun, and the lithium destruction of a solar model (dashed line) are indicated.

Table 2. Mass and age determinations from TGEC models compared to observations of (a) Takeda et al. (2007) and (b) Meléndez \& Ramírez (2007).

\begin{tabular}{ccccccc}
\hline \hline Name & \multicolumn{3}{c}{ Mass $\left[M_{\odot}\right]$} & \multicolumn{3}{c}{ Age [Gyr] } \\
& (a) & (b) & (TGEC) & (a) & (b) & (TGEC) \\
\hline HIP 55 459 & $1.03_{-0.04}^{+0.05}$ & & $1.018 \pm 0.007$ & $3.80_{-2.10}^{+2.51}$ & & $4.27_{-1.32}^{+1.94}$ \\
& & & & & $4.7 \pm 1.0$ & $5.77_{0.24}^{+0.81}$ \\
(HD 98 618) & & $1.03 \pm 0.03$ & $1.013 \pm 0.005$ & & & $2.89_{-0.81}^{+1.09}$ \\
& & & & & & \\
HIP 79 672 & $1.02_{-0.02}^{+0.03}$ & & $1.007 \pm 0.006$ & $4.57_{-2.87}^{+0.33}$ & & \\
(18 Sco) & & $1.04 \pm 0.03$ & $1.015 \pm 0.06$ & & $3.4 \pm 1.0$ & $5.03_{-1.29}^{+1.25}$ \\
HIP 100 963 & $1.00_{-0.02}^{+0.03}$ & & $0.998 \pm 0.006$ & $5.13_{-2.99}^{+0.00}$ & & $3.07_{-1.06}^{+0.73}$ \\
HIP 56 948 & & $1.00 \pm 0.03$ & $0.994 \pm 0.004$ & & $5.8 \pm 1.0$ & $4.71_{-1.23}^{+1.39}$ \\
HIP 73 815 & & $1.00 \pm 0.03$ & $0.992 \pm 0.005$ & & $6.3 \pm 1.0$ & $5.76_{-0.79}^{+1.13}$ \\
\hline
\end{tabular}

\section{Conclusions}

Although a fundamental parameter in studies of stellar evolution, the mass of single stars cannot be derived directly from observations. The mass is a crucial parameter in characterizing a solar twin. We have analyzed the 5 most probable solar twins selected from the literature and developed a method constrained by the $\mathrm{Li}$ abundance observations to determine the mass and age of the stars. Our mass determination is based on the Li depletion in low-mass stars being strongly mass dependent. The Li depletion in solar-type stars is caused by the microscopic diffusion and the rotation-induced mixing. Lithium atoms diffuse below the outer convective zone and if this zone is deep enough, the meridional circulation transports them from the bottom of the convective zone to the destruction layers. This process provides an explanation of the low Li abundance observed in the Sun and some solar twins. We used accurate lithium abundance observations compared with non-standard models to provide precise information 

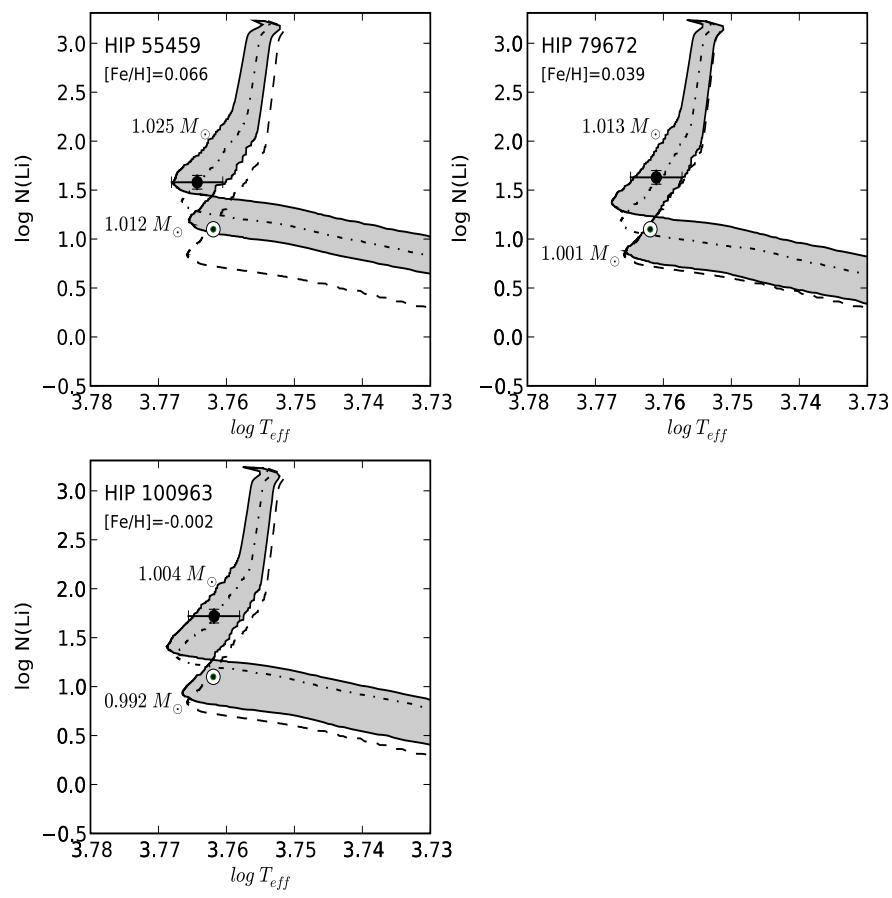

Fig. 4. Solar twins observed by Takeda et al. (2007), the caption is as in Fig. 3.
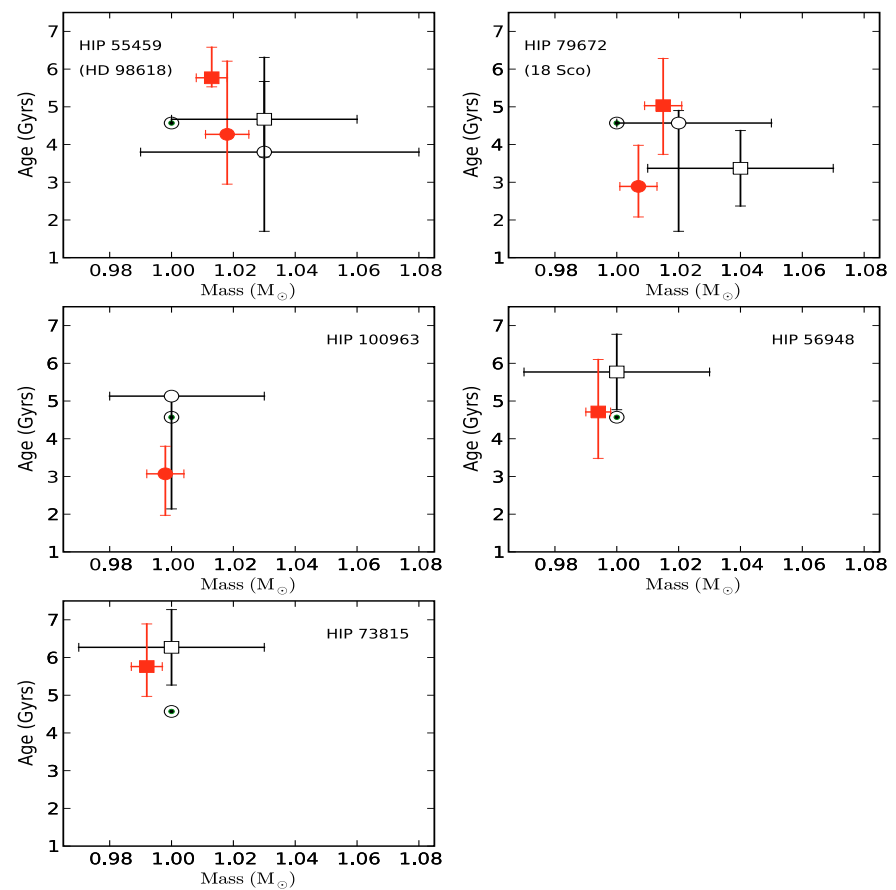

Fig. 5. Comparison between masses and ages determined by TGEC models (filled symbols) and masses and ages estimated by the observations (open symbols). Squares correspond to the solar twins observed by Mélendez \& Ramírez (2007) and circles to the solar twins observed by Takeda et al. (2007). The errors bars are as described in the text.

about the age and mass of these stars, which are more accurately determined than by classical methods. For each solar twin, we computed the non-standard model that gives the best-fit solution to the observed lithium abundance. For HIP 56948, our analysis infers a mass of $0.994 \pm 0.004 M_{\odot}$ and age of $4.71 \pm 1.39$ Gyr. We have confirmed that HIP 56948 is the most likely solar twin at the present time, its $\mathrm{Li}$ abundance being very close to the solar value. HIP 73815 also has a low $\mathrm{Li}$ abundance and was proposed by Meléndez \& Ramírez (2007) as an excellent solartwin candidate, although our analysis, constrained by its Li abundance, infers a star slightly less massive and older than the Sun. HIP 55459 and 18 Sco are found to be slightly more massive than the Sun, which is consistent with their high Li abundance. HIP 100963 seems to be a young solar twin.

In summary, we have shown that an accurate lithium abundance measurement and non-standard models provide more precise information about the age and mass of solar twins, more precisely than determined by classical methods. This demonstrates that the physical properties of the stellar interior should be taken into consideration when attempting a more realistic characterization of a solar twin star.

Acknowledgements. This research has made use of SIMBAD and VIZIER databases, operated at CDS (Strasbourg, France). JRM thanks support from the FCT (Ciencia 2007). Research activities of the Stellar Board at the Federal University of Rio Grande do Norte are supported by continuous grants from CNPq and FAPERN Brazilian Agencies. GFPM acknowledges financial support by CNPq and FAPERJ. The authors acknowledge support from the FCT/CAPES cooperation agreement No. 237/09.

\section{References}

Alexander, D. R., \& Fergusson, J. W. 1994, ApJ, 437, 879

Angulo, C., Arnould, M., \& Rayet, M. 1999, Nucl. Phys. A, 656, 1

Asplund, M., Grevesse, N., \& Sauval, A. J. 2005, in Cosmic Abundances as Records of Stellar Evolution and Nucleosynthesis, ed. T. G. Barnes III, \& F. N. Bash, ASP Conf. Ser., 336, 25

Bahcall, J. N., \& Pinsonneault, M. H. 1992, RvMP, 64, 885

Balachandran, S. C., \& Bell, R. A. 1998, Nature, 392, 791

Basu, S., Christensen-Dalsgaard, J., Chaplin, W. J., et al. 1997, MNRAS, 292, 243

Böhm-Vitense, E. 1958, ZAp, 46, 108

Brun, A. S., Turck-Chièze, S., \& Zahn, J.-P. 1998, in Structure and Dynamics of the Interior of the Sun and Sun-like Stars, ESA Publications Division, SP-418, 439

Brun, A. S., Turck-Chièze, S., \& Zahn, J.-P. 1999, ApJ, 525, 1032

Caffau, E., Maiorca, E., Bonifacio, P., et al. 2009, A\&A, 498, 877

Cayrel de Strobel, G. 1996, A\&ARv, 7, 243

Chaboyer, B., Demarque, P., \& Pinsonneault, M. H. 1995, ApJ, 441, 865

Charbonnel, C., \& do Nascimento, J. D., Jr. 1998, A\&A, 336, 915

Charbonnel, C., \& Talon, S. 1999, A\&A, 351, 635

Charbonnel, C., \& Talon, S. 2005, Science, 309, 2189

Delahaye, F., \& Pinsonneault, M. H. 2005, ApJ, 649, 529

Deliyannis, C. P., \& Pinsonneault, M. 1997, ApJ, 488, 833

do Nascimento, J. D., Jr., Charbonnel, C., Lebre, A., de Laverny, P., \& De Medeiros, J. R. 2000, A\&A, 357, 931

García López, R. J., \& Spruit, H. C. 1991, ApJ, 377, 268

Gough, D., \& McIntyre, M. E. 1998, Nature, 394, 755

Grevesse, N., \& Noels, A. 1993, in Origin and evolution of the elements: proceedings of a symposium in honour of H. Reeves, Paris, June 22-25, 1992, ed. N. Prantzos, E. Vangioni-Flam, \& M. Casse (Cambridge, England: Cambridge University Press), 14

Grevesse, N., \& Sauval, A. J. 1998, Space Sci. Rev., 85, 161

Holmberg, J., Nordström, B., \& Andersen, J. 2007, A\&A, 475, 519

Hui-Bon-Hoa, A. 2008, Ap\&SS, 316, 55

Iglesias, C. A., \& Rogers, F. J. 1996, ApJ, 464, 943

Izumiura, H. 1999, in Proc. 4th East Asian Meeting on Astronomy, Observational Astrophysics in Asia and its Future ed. P. S. Chen (Kunming: Yunnan Observatory), 77

Lambert, D. L., \& Reddy, B. E. 2004, MNRAS, 349, 757

Luck, R. E., \& Heiter, U. 2005, AJ, 129, 1063

Maeder, A., \& Zahn, J.-P 1998, A\&A, 334, 1000

Masana, E., Jordi, C., \& Ribas, I. 2006, A\&A, 450, 735

Meléndez, J., \& Ramírez, I. 2007, ApJ, 669, L89

Meléndez, J., Dodds-Eden, K., \& Robles, J. A. 2006, ApJ, 641, 133

Michaud, G. 1986, ApJ, 302, 650

Montalbán, J., \& Schatzmann, E. 2000, A\&A, 354, 943

Palacios, A., Talon, S., Charbonnel, C., \& Forestini, M. 2003, A\&A, 399, 603

Paquette, C., Pelletier, C., Fontaine, G., \& Michaud, G. 1986, ApJS, 61, 177 
Pasquini, L., Biazzo, K., Bonifacio, P., Randich, S., \& Bedin, L. R. 2008, A\&A, 489, 677

Piau, L., Randich, S., \& Palla, F. 2003, A\&A, 408, 1037

Pinsonneault, M. H., Deliyannis, C. P., \& Demarque, P. 1992, ApJS, 78, 179

Porto de Mello, G. F., \& da Silva, L. 1997, ApJ, 482, L89

Richard, O., Vauclair, S., Charbonnel, C., \& Dziembowski, W. A. 1996, A\&A, 312,1000

Richard, O., Théado, S., \& Vauclair, S. 2004, SoPh, 220, 243

Robinson, S. E., Ammons, S. M., Kretke, K. A., et al. 2007, ApJS, 169, 430

Rogers, F. J., \& Nayfonov, A. 2002, ApJ, 576, 1064

Schatzman, E., \& Baglin, A. 1991, A\&A, 249, 125

Skumanich, A. 1972, ApJ, 171, 565

Spiegel, E. A., \& Zahn, J.-P. 1992, A\&A, 265, 106

Swenson, F. J., \& Faulkner, J. 1992, ApJ, 395, 654

Takeda, Y., Kawanomoto, S., Honda, S., Ando, H., \& Sakurai, T. 2007, A\&A, 468, 663
Takeda, Y., \& Tajitsu, A. 2009 [arXiv: 0901.2509]

Talon, S., \& Charbonnel, C. 1998, A\&A, 335, 959

Talon, S., \& Charbonnel, C. 2005, A\&A, 440, 981

Théado, S., \& Vauclair, S. 2003a, ApJ, 587, 784

Théado, S., \& Vauclair, S. 2003b, ApJ, 587, 795

Tull, R. G., MacQueen, P. J., Sneden, C., \& Lambert, D. L. 1995, PASP, 107, 251

Turcotte, S., Richer, J., Michaud, G., Iglesias, C. A., \& Rogers, F. J. 1998, ApJ, 504,539

Valenti, J. A., \& Fisher, D. A. 2005, ApJS, 159, 141

VandenBerg, D. A., Edvardsson, B., Eriksson, K., \& Gustafsson, B. 2008, ApJ, 675,746

van Leeuwen, F. 2007, ASSL, 350

Vauclair, S. 1991, Proceeding of the 14th Symp. Int. Astron. Union, 145, 32

Vauclair, S., \& Théado, S. 2003 ApJ, 587, 777

Zahn, J.-P. 1992, A\&A, 265, 115 